\title{
Charmed hadron properties and spectroscopy at LHCb
}

\author{
Yixiong Zhou ${ }^{a, *}$ \\ on behalf of the LHCb collaboration \\ ${ }^{a}$ University of Chinese Academy of Sciences, \\ No.19(A) Yuquan Road, Beijing, China
}

E-mail: zhouyixiong@mails.ucas.ac.cn

We report new measurements relative to charmed hadron spectroscopy and measurement of charm hadron properties by the LHCb collaboration. Two new $\Xi_{c}^{0}$ baryons are observed, and the lifetimes of the $\Lambda_{c}^{+}, \Xi_{c}^{0}$ and $\Xi_{c}^{+}$baryons are measured with greatly improved precision compared to the current world average. The doubly Cabibbo-suppressed decays of $\Xi_{c}^{+} \rightarrow p \phi$ and $\Xi_{c}^{0} \rightarrow \Lambda_{c}^{+} \pi^{-}$are observed for the first time. The most precise measurement of $\Xi_{c c}^{++}$mass and production ratio is performed. Finally, the $\Xi_{c c}^{+}$baryon is searched for in the $\Lambda_{c}^{+} K^{-} \pi^{+}$final state.

40th International Conference on High Energy Physics - ICHEP2020

July 28 - August 6, 2020

Prague, Czech Republic (virtual meeting)

${ }^{*}$ Speaker 


\section{Introduction}

The measurement of the properties of heavy-flavoured hadrons is essential to test various theoretical approaches, and therefore deepen our understanding of non-perturbative quantum chromodynamics (QCD). In this proceeding, seven new measurements from the LHCb collaboration are reported.

\section{Observation of new $\Xi_{c}^{0}$ baryons decaying to $\Lambda_{c}^{+} K^{-}$}

Singly charmed baryons are composed of a charm quark and two light quarks. They provide an excellent laboratory to test various theoretical models, in which the three constituent quarks are effectively described in terms of a heavy quark plus a light diquark system [1]. In 2017, the LHCb collaboration reported the observation of five new narrow $\Omega_{c}^{0}$ baryons decaying to the $\Xi_{c}^{+} K^{-}$ final state [2]. It is currently not understood why the natural widths of these resonances are small. Investigating a different charmed mass spectrum could lead to a better understanding of this feature.

The $\Lambda_{c}^{+}\left(\rightarrow p K^{-} \pi^{+}\right) K^{-}$mass spectrum is studied with LHCb data collected at a centre-ofmass energy of $13 \mathrm{TeV}$, corresponding to an integrated luminosity of $5.6 \mathrm{fb}^{-1}$. Three narrow structures are observed in the $\Lambda_{c}^{+} K^{-}$candidate spectrum, hereafter named $\Xi_{c}(2923)^{0}, \Xi_{c}(2939)^{0}$ and $\Xi_{c}(2965)^{0}$ [3]. The measured masses and natural widths of $\Xi_{c}(2923)^{0}, \Xi_{c}(2939)^{0}$ and $\Xi_{c}(2965)^{0}$ are summarised in Table 1 . The $\Xi_{c}(2923)^{0}$ and $\Xi_{c}(2939)^{0}$ baryons are new states. The $\Xi_{c}(2965)^{0}$ state is in the vicinity of the known $\Xi_{c}(2970)^{0}$ baryon [4], however, their masses and natural widths differ significantly.

Table 1: Summary of the measured masses and the natural widths, where the first uncertainty is statistical and the second uncertainty is systematic.

\begin{tabular}{ccr}
\hline Resonance & Mass $\left[\mathrm{MeV} / c^{2}\right]$ & $\Gamma\left[\mathrm{MeV} / c^{2}\right]$ \\
\hline$\Xi_{c}(2923)^{0}$ & $2923.04 \pm 0.25 \pm 0.24$ & $7.1 \pm 0.8 \pm 1.8$ \\
$\Xi_{c}(2939)^{0}$ & $2938.55 \pm 0.21 \pm 0.22$ & $10.2 \pm 0.8 \pm 1.1$ \\
$\Xi_{c}(2965)^{0}$ & $2964.88 \pm 0.26 \pm 0.20$ & $14.1 \pm 0.9 \pm 1.3$ \\
\hline
\end{tabular}

\section{First branching fraction measurement of the suppressed decay $\Xi_{c}^{0} \rightarrow \Lambda_{c}^{+} \pi^{-}$}

The $\Xi_{c}^{0} \rightarrow \Lambda_{c}^{+} \pi^{-}$decay proceeds through $s \rightarrow u(\bar{u} d)$ and $c s \rightarrow d c$ (weak scattering) processes. Studies of such decay will help us to understand the underlying dynamics of charmed baryon decays and distinguish between different theoretical models. The branching fraction of $\Xi_{c}^{0} \rightarrow \Lambda_{c}^{+} \pi^{-}$has not been previously measured.

The branching fraction $\mathcal{B}\left(\Xi_{c}^{0} \rightarrow \Lambda_{c}^{+} \pi^{-}\right)$is measured with LHCb data collected at a centreof-mass energy of $13 \mathrm{TeV}$, corresponding to an integrated luminosity of $3.8 \mathrm{fb}^{-1}$. To calculate the branching fraction for $\Xi_{c}^{0} \rightarrow \Lambda_{c}^{+} \pi^{-}$, two normalisation methods are used. The first normalisation method uses the heavy-quark symmetry, where the production ratio of $\Xi_{c}^{0}$ to $\Lambda_{c}^{+}$baryons $f_{\Xi_{c}^{0}} / f_{\Lambda_{c}^{+}}$is estimated as $C \cdot f_{\Xi_{b}^{-}} / f_{\Lambda_{b}^{0}}$, where $C$ is a correction factor for feed-downs of excited $\Xi_{b}$ baryons that do not have equal rates to $\Xi_{b}^{-}$and $\Xi_{b}^{0}$ final states. The second method uses the branching fraction 
of $\Xi_{c}^{+} \rightarrow p K^{-} \pi^{+}$measured by Belle [5]. Taking the weighted average value of the two methods, the branching fraction of $\Xi_{c}^{0} \rightarrow \Lambda_{c}^{+} \pi^{-}$is determined to be $\mathcal{B}\left(\Xi_{c}^{0} \rightarrow \Lambda_{c}^{+} \pi^{-}\right)=(0.55 \pm 0.02$ (stat) \pm 0.18 (syst) $) \%$. The $\mathcal{B}\left(\Xi_{c}^{+} \rightarrow p K^{-} \pi^{+}\right)$is determined to be $(1.135 \pm 0.002$ (stat) \pm 0.387 (syst) $) \%$ using $\mathcal{B}\left(\Lambda_{c}^{+} \rightarrow p K^{-} \pi^{+}\right)$as normalisation [6].

\section{Observation of doubly Cabibbo-suppressed decay $\Xi_{c}^{+} \rightarrow p \phi$}

A systematic study of the relative contributions of doubly Cabibbo-suppressed (DCS) and Cabibbo-favoured process to decays of charm baryons could shed light onto the role of the nonspectator quark, and in particular Pauli interference [7]. Such studies would be helpful for a better understanding of the lifetime hierarchy of charm baryons. So far only one DCS charm-baryon decay, $\Lambda_{c}^{+} \rightarrow p K^{+} \pi^{-}$, has been observed [8,9].

In this work the ratio of branching fractions between the $\Xi_{c}^{+} \rightarrow p \phi$ and $\Xi_{c}^{+} \rightarrow p K^{-} \pi^{+}$decay is measured with $\mathrm{LHCb}$ data collected at a centre-of-mass energy of $8 \mathrm{TeV}$, corresponding to an integrated luminosity of $2 \mathrm{fb}^{-1}$. To determine the signal yield of $\Xi_{c}^{+} \rightarrow p \phi$ decay, a two-dimensional unbinned extended maximum likelihood fit to the $m_{p K^{+} K^{-}}$and $m_{K^{+} K^{-}}$distributions is performed. The resulting relative branching fraction ratio with respect to the singly Cabibbo-suppressed $\Xi_{c}^{+} \rightarrow$ $p K^{-} \pi^{+}$decay channel is measured to be $R_{p \phi}=(19.8 \pm 0.7$ (stat) \pm 0.9 (syst) $) \times 10^{-3}$ [10]. The DCS $\Xi_{c}^{+} \rightarrow p \phi$ decay is observed for the first time, and an evidence at the level of $3.5 \sigma$ is found for a non- $\phi$ contribution to the $\Xi_{c}^{+} \rightarrow p K^{+} K^{-}$decay.

\section{Precision measurement of the $\Lambda_{c}^{+}, \Xi_{c}^{+}$and $\Xi_{c}^{0}$ baryon lifetimes}

Precision measurement of the baryons lifetime allows stringent test of their association theoretical predictions. Recently the LHCb collaboration reported a measurement of the $\Omega_{c}^{0}$ lifetime that was nearly four times larger than, and inconsistent with, the world average value [11]. Given the overall relatively poor precision on the $\Lambda_{c}^{+}, \Xi_{c}^{+}$and $\Xi_{c}^{0}$ lifetimes compared to those of the charm mesons, it is important to have additional precise measurements of the lifetimes of these baryons.

Using a similar approach as the $\Omega_{c}^{0}$ lifetime measurement, the lifetimes are measured using samples of semileptonic $H_{b} \rightarrow H_{c} \mu v_{\mu} X$ decays, where $H_{b}$ represents a $\Lambda_{b}^{0}, \Xi_{b}^{0}$ or $\Xi_{b}^{-}$baryon and $H_{c}$ corresponds to a $\Lambda_{c}^{+}, \Xi_{c}^{+}$or $\Xi_{c}^{0}$, respectively, $X$ standing for any additional undetected particles. To reduce the systematic uncertainties, the ratio of the lifetime relative to that of the $D^{+}$meson $r_{H_{c}} \equiv \frac{\tau_{H_{c}}}{\tau_{D^{+}}}$is determined from a simultaneous fit to the $H_{c}$ decay-time spectrum and to that of the $D^{+}$meson. Multiplying these ratios by the $D^{+}$lifetime leads to

$$
\begin{aligned}
& \tau_{\Lambda_{c}^{+}}=203.5 \pm 1.0 \pm 1.9 \mathrm{fs}, \\
& \tau_{\Xi_{c}^{+}}=456.8 \pm 3.5 \pm 4.2 \mathrm{fs}, \\
& \tau_{\Xi_{c}^{0}}=154.5 \pm 1.7 \pm 1.9 \mathrm{fs},
\end{aligned}
$$

where the first uncertainty is statistical and the second is systematic [12]. The $\Lambda_{c}^{+}$and $\Xi_{c}^{+}$lifetimes are measured with about $1 \%$ precision and are consistent with the existing world averages. The $\Xi_{c}^{0}$ lifetime is measured with about $1.8 \%$ precision, and is $3.3 \sigma$ larger than the world average value of 
$112_{-10}^{+13} \mathrm{fs}$. The uncertainties on these measurements are on average 3-4 times smaller than those of the existing world average, and have precision comparable to that achieved for charm mesons.

\section{Precision measurement of the $\Xi_{c c}^{++}$mass}

The doubly charmed baryon $\Xi_{c c}^{++}(c c u)$ was first observed by the LHCb collaboration in 2017 via the $\Xi_{c c}^{++} \rightarrow \Lambda_{c}^{+} K^{-} \pi^{+} \pi^{+}$decay channel, with $\Lambda_{c}^{+}$decaying to the $p K^{-} \pi^{+}$final state [13]. This observation was then confirmed in another decay channel, $\Xi_{c c}^{++} \rightarrow \Xi_{c}^{+} \pi^{+}$with $\Xi_{c}^{+}$decaying to $p K^{-} \pi^{+}$final state [14]. The $\Xi_{c c}^{++}$mass was measured to be $3621.24 \pm 0.65$ (stat) \pm 0.31 (syst) $\mathrm{MeV} / \mathrm{c}^{2}$. Theoretical calculations of the $\Xi_{c c}^{++}$mass after the LHCb observation fall into a $\pm 20 \mathrm{MeV} / c^{2}$ window around the experimental value measured by LHCb [15]. At present, experimental uncertainty on the $\Xi_{c c}^{++}$mass is still large compared to that of the singly charmed baryons.

In this work, the $\Xi_{c c}^{++}$mass was measured using the $\Xi_{c c}^{++} \rightarrow \Lambda_{c}^{+}\left(\rightarrow p K^{-} \pi^{+}\right) K^{-} \pi^{+} \pi^{+}$and $\Xi_{c c}^{++} \rightarrow \Xi_{c}^{+}\left(\rightarrow p K^{-} \pi^{+}\right) \pi^{+}$decay modes. The analysis uses a data sample corresponding to an integrated luminosity of $5.6 \mathrm{fb}^{-1}$, collected by the LHCb experiment at a centre-of-mass energy of $13 \mathrm{TeV}$. The resulting values of the $\Xi_{c c}^{++}$mass using the $\Xi_{c c}^{++} \rightarrow \Lambda_{c}^{+} K^{-} \pi^{+} \pi^{+}$and $\Xi_{c c}^{++} \rightarrow \Xi_{c}^{+} \pi^{+}$decay modes are $3621.53 \pm 0.24$ (stat) \pm 0.29 (syst) $\mathrm{MeV} / \mathrm{c}^{2}$, and $3621.95 \pm 0.60$ (stat) \pm 0.49 (syst) $\mathrm{MeV} / \mathrm{c}^{2}$, respectively. By combining these two measurements the $\Xi_{c c}^{++}$mass is determined to be $3621.55 \pm 0.23$ (stat) \pm 0.30 (syst) $\mathrm{MeV} / c^{2}$ [16]. This is the most precise measurement of the $\Xi_{c c}^{++}$mass to date.

\section{Measurement of $\Xi_{c c}^{++}$production in $p p$ collisions at $\sqrt{s}=13 \mathrm{TeV}$}

Baryons containing two charm quarks and a light quark provide a unique system for testing the low-energy limit of QCD. The production cross-section of doubly charmed baryons in $p p$ collisions at a centre-of-mass energy $\sqrt{s}=13 \mathrm{TeV}$ is predicted to be in $60-1800 \mathrm{nb}$ range, which is between $10^{-4}$ and $10^{-3}$ times that of the total charm production [17].

The production of $\Xi_{c c}^{++}$in $p p$ collisions at a centre-of-mass energy $\sqrt{s}=13 \mathrm{TeV}$ is measured by $\mathrm{LHCb}$, with a dataset corresponding to an integrated luminosity of $1.7 \mathrm{fb}^{-1}$. The production cross-section, $\sigma\left(\Xi_{c c}^{+}\right)$, times the branching fraction of the $\Xi_{c c}^{++} \rightarrow \Lambda_{c}^{+} K^{-} \pi^{+} \pi^{+}$decay, is measured relative to the prompt $\Lambda_{c}^{+}$production cross-section, $\sigma\left(\Lambda_{c}^{+}\right)$, in the transverse momentum range $4<p_{\mathrm{T}}<15 \mathrm{GeV} / c$ and the rapidity range $2.0<y<4.5$. The production ratio is defined as

$$
R \equiv \frac{\sigma\left(\Xi_{c c}^{++}\right) \times \mathcal{B}\left(\Xi_{c c}^{++} \rightarrow \Lambda_{c}^{+} K^{-} \pi^{+} \pi^{+}\right)}{\sigma\left(\Lambda_{c}^{+}\right)} .
$$

The production ratio is measured to be $(2.22 \pm 0.27$ (stat) \pm 0.29 (syst) $) \times 10^{-4}$ [18], assuming the central value of the $\Xi_{c c}^{++}$lifetime measured in Ref. [19]. This is the first measurement of the production of the doubly charmed baryons in $p p$ collisions.

\section{Search for the doubly charmed baryon $\Xi_{c c}^{+}$}

The doubly charmed baryon $\Xi_{c c}^{+}$was first reported by the SELEX collaboration with its decays into $\Lambda_{c}^{+} K^{-} \pi^{+}$and $p D^{+} K^{-}$[20, 21]. Searches in different environments by the FOCUS [22], Barbar [23], LHCb [24] and Belle [25] experiments did not confirm the SELEX results. 
In this work, we use full $p p$ collision data recorded with the LHCb detector corresponding to a total integrated luminosity about $9 \mathrm{fb}^{-1}$. This data sample is about ten times larger than that of the previous $\Xi_{c c}^{+}$search by the LHCb collaboration using only 2011 data [24]. The largest local significance is about $3.1 \sigma$ in region around $3620 \mathrm{MeV} / \mathrm{c}^{2}$. Taking into account the look-elsewhere effect the global significance is about $1.7 \sigma$. Upper limits are set at $95 \%$ credibility level on the ratio of the $\Xi_{c c}^{++}$production cross-section times the branching fraction to that of the $\Lambda_{c}^{+}\left(R\left(\Lambda_{c}^{+}\right)\right)$and $\Xi_{c c}^{++}\left(R\left(\Xi_{c c}^{++}\right)\right)$baryons. The limits are determined as functions of the $\Xi_{c c}^{+}$mass for different lifetime hypotheses, in the rapidity range from 2.0 to 4.5 and the transverse momentum range from 4 to 15 $\mathrm{GeV} / c$. The upper limit on the production ratio $R\left(\Lambda_{c}^{+}\right)\left(R\left(\Xi_{c c}^{++}\right)\right)$depends strongly on the considered mass and lifetime of the $\Xi_{c c}^{+}$baryon, varying from $0.45 \times 10^{-3}(2.0)$ for 40 fs to $0.12 \times 10^{-3}(0.5)$ for $160 \mathrm{fs}$ [26]. The upper limits on $R\left(\Lambda_{c}^{+}\right)$are improved by order of magnitude compared to the previous LHCb search and are significantly below the value reported by SELEX, albeit in a different production environment.

\section{Summary}

Seven new results on the charmed hadron properties and spectroscopy at LHCb are reported. The observation of two new $\Xi_{c}^{0}$ states [3], and the lifetime of $\Lambda_{c}^{+}, \Xi_{c}^{0}$ and $\Xi_{c}^{+}$are measured with significantly improved precision [12], the first observation of suppressed decays of $\Xi_{c}^{+} \rightarrow p \phi$ and $\Xi_{c}^{0} \rightarrow \Lambda_{c}^{+} \pi^{-}[6,10]$, and the most precise measurement of $\Xi_{c c}^{++}$mass and production ratio $[16,18]$, and last is the search of $\Xi_{c c}^{+}$[26]. More results of charm spectroscopy at LHCb are expected soon.

\section{References}

[1] A.G. Grozin. Introduction to the heavy quark effective theory. part 1. 121992.

[2] Roel Aaij et al. Observation of five new narrow $\Omega_{c}^{0}$ states decaying to $\Xi_{c}^{+} K^{-}$. Phys. Rev. Lett., 118(18):182001, 2017.

[3] Roel Aaij et al. Observation of new $\Xi_{c}^{0}$ baryons decaying to $\Lambda_{c}^{+} K^{-}$. Phys. Rev. Lett., 124(22):222001, 2020.

[4] P.A. Zyla et al. Review of particle physics. PTEP, 2020(8):083C01, 2020.

[5] Y.B. Li et al. First measurements of absolute branching fractions of the $\Xi_{c}^{+}$baryon at Belle. Phys. Rev. D, 100(3):031101, 2019.

[6] Roel Aaij et al. First branching fraction measurement of the suppressed decay $\Xi_{c}^{0} \rightarrow \pi^{-} \Lambda_{c}^{+}$. Phys. Rev. D, 102(7):071101, 2020.

[7] S. Bianco, F.L. Fabbri, D. Benson, and I. Bigi. A Cicerone for the physics of charm. Riv. Nuovo Cim., 26N7:1-200, 2003.

[8] S.B. Yang et al. First observation of doubly Cabibbo-Suppressed decay of a charmed baryon: $\Lambda_{c}^{+} \rightarrow p K^{+} \pi^{-}$. Phys. Rev. Lett., 117(1):011801, 2016. 
[9] Roel Aaij et al. Measurements of the branching fractions of $\Lambda_{c}^{+} \rightarrow p \pi^{-} \pi^{+}, \Lambda_{c}^{+} \rightarrow p K^{-} K^{+}$, and $\Lambda_{c}^{+} \rightarrow p \pi^{-} K^{+} . J H E P, 03: 043,2018$.

[10] Roel Aaij et al. Observation of the doubly Cabibbo-suppressed decay $\Xi_{c}^{+} \rightarrow p \phi$. JHEP, 04:084, 2019.

[11] Roel Aaij et al. Measurement of the $\Omega_{c}^{0}$ baryon lifetime. Phys. Rev. Lett., 121(9):092003, 2018.

[12] Roel Aaij et al. Precision measurement of the $\Lambda_{c}^{+}$, $\Xi_{c}^{+}$and $\Xi_{c}^{0}$ baryon lifetimes. Phys. Rev. D, 100(3):032001, 2019.

[13] Roel Aaij et al. Observation of the doubly charmed baryon $\Xi_{c c}^{++}$. Phys. Rev. Lett., 119(11):112001, 2017.

[14] Roel Aaij et al. First observation of the doubly charmed baryon decay $\Xi_{c c}^{++} \rightarrow \Xi_{c}^{+} \pi^{+}$. Phys. Rev. Lett., 121(16):162002, 2018.

[15] Xin-Zhen Weng, Xiao-Lin Chen, and Wei-Zhen Deng. Masses of doubly heavy-quark baryons in an extended chromomagnetic model. Phys. Rev. D, 97(5):054008, 2018.

[16] Roel Aaij et al. Precision measurement of the $\Xi_{c c}^{++}$mass. JHEP, 02:049, 2020.

[17] V.V. Kiselev and A.K. Likhoded. Baryons with two heavy quarks. Phys. Usp., 45:455-506, 2002.

[18] Roel Aaij et al. Measurement of $\Xi_{c c}^{++}$production in $p p$ collisions at $\sqrt{s}=13 \mathrm{TeV}$. Chin. Phys. C, 44(2):022001, 2020.

[19] Roel Aaij et al. Measurement of the lifetime of the doubly charmed baryon $\Xi_{c c}^{++}$. Phys. Rev. Lett., 121(5):052002, 2018.

[20] M. Mattson et al. First observation of the doubly charmed baryon $\Xi_{c c}^{+}$. Phys. Rev. Lett., 89:112001, 2002.

[21] A. Ocherashvili et al. Confirmation of the double charm baryon $\Xi_{c c}^{+}(3520)$ via its decay to $p D^{+} K^{-}$. Phys. Lett. B, 628:18-24, 2005.

[22] Sergio P. Ratti et al. New results on $c$-baryons and a search for $c c$-baryons in focus. Nuclear Physics B - Proceedings Supplements, 115:33 - 36, 2003. Hyperons, Charm and Beauty Hadrons.

[23] Bernard Aubert et al. Search for doubly charmed baryons $\Xi_{c c}^{+}$and $\Xi_{c c}^{++}$in BABAR. Phys. Rev. D, 74:011103, 2006.

[24] R Aaij et al. Search for the doubly charmed baryon $\Xi_{c c}^{+}$. JHEP, 12:090, 2013.

[25] Y. Kato et al. Search for doubly charmed baryons and study of charmed strange baryons at Belle. Phys. Rev. D, 89(5):052003, 2014.

[26] Roel Aaij et al. Search for the doubly charmed baryon $\Xi_{c c}^{+}$. Sci. China Phys. Mech. Astron., 63(2):221062, 2020. 